\title{
THE OPTIMISING FORCE BALANCE EXERCISED IN THE WHEEL - PROFILE CONTACT FORCE DURING THE CURVED PATH. AN EXPERIMENTAL APPROACH OF USING CURVILINEAR PROFILES
}

\author{
Koci Doraci, Alfred Hasanaj*
}

University Polytechnic of Tirana, Tirana, Albania

\begin{abstract}
This paper's objective is to contribute to the optimization of the problem of guiding forces during the movement of a railway vehicle. The paper proposes an innovative solution as compared to classical authors' suggestions, which are limited to a small number of alternatives. The methodology follows an experimental approach. A two-stage experiment is performed where experimental conditions are modeled after profiles designed following a curved path with a radius of $500 \mathrm{~m}$ (by definition classified as tight curve). Standard profiles were used in the first experimental stage used, while curvilinear profiles were exploited the second experimental stage. After the experiment was conducted, data concerning (1) displacement (2) moments of force and (3) guiding forces of wheelrail contact were analyzed and compared for both stages: both standard profiles and curvilinear profiles. After considering the experimental results, the major conclusions of the paper are: (1) in the case of curvilinear profiles, small movements of the vehicle lead to a change in the size of the wheel displacement smaller than the corresponding change in standard profiles; (2) moments of force are greater in the case of standard profiles compared to curvilinear ones; and (3) curvilinear profiles enable movement without many contact points with the wheel, friction forces exert their action in longitudinal direction, thus causing a smaller value of guiding forces.
\end{abstract}

Key words: Guiding forces, curvilinear profile, moment of forces, wheel displacement

DOI: $10.21175 /$ RadProc.2017.60

\section{PROBLEM DEFINITION}

Firstly, it is important to define the meaning of "guiding forces during the movement of the railway vehicle".

According to [1], the guiding force is the total of dynamic characteristics that emerges during the movement of the railway vehicle through curves. Apart from [1], [2] states that these forces emerge on each wheel during their contact with rail profiles along curves and the most important influences of this contact are: (1) wheels wear and (2) rails wear.

The two major components of these guiding forces are: (1) the dynamic component and (2) the static (quasi-static) component.

It has already been tested and approved that dynamic guiding forces cause an increase in the wear index of both the wheel and the rail, especially when the vehicle crosses a curved rail section. Moreover, the stronger the curve, the higher the dynamic guiding forces, and, as a consequence, the higher the wheel and profile wear [3]. A strong curve is defined as a curve which can be classified as a small radius curve, usually below the curve radius interval of 500-650 meters [4].
Given the above, it can be concluded that the movement of a vehicle along a curved rail can be considered sustainable only when the quasi-static component of the lateral forces of interaction of wheels with rails significantly exceeds the value of dynamic forces.

Therefore, the problem that this paper addresses can be formulated as follows: minimizing dynamic guiding forces that emerge during the interaction of the train wheel and profile in curves, especially in low radius curves (strong rail curves).

\subsection{Paper's objective}

The purpose of this paper is to determine an alternative for reducing, or even minimizing, driving forces, which, as explained in the problem identification section, emerge when a train vehicle passes over low radius curves.

Of course, there are many different referencing systems according to which this problem may be analyzed; however, it is inevitable that resolving the train driving force in tight curves be accompanied by reducing wheel and/ or profile wear.

This paper's objective is heavily conditioned by the formulation of the driving force problem. 
Taking into account the methodology of designing a solution model for the problem of the train driving force in tight curves, it should be considered that the following conditions have guided this design:

- This paper addresses the reduction of the train driving force in tight curves as a problem of reducing its dynamic component (non-static or quasi-static force component)

- This paper focuses on the driving force on the horizontal (longitudinal) plane and on vertical (lateral) surfaces.

- The optimization object in this paper will be the reduction of profile wear.

Having in mind these two conditions and restrictions on perceiving the train driving force problem, it can be finally reformulated that this paper's objective is to propose a technical solution that will achieve sustainable movement of the train vehicle in low radius curves by reducing dynamic forces which prevent wheel rolling with regard to the horizontal plane and profile consummation (profile wear).

\subsection{Literature Review}

The problem of addressing static guiding force reduction during train wheel and profile contact when the vehicle crosses a strong curve has been explored by a large number of authors. Consequently, multiple solutions have been proposed.

The latest paper that addresses this problem is [5] According to these authors, optimization is achieved in terms of minimizing rail wear, which can be achieved by implementing a genetic algorithm encompassing the relationship between metal loss from the vehicle and coordinates on the rail.

Before this work, many other authors have proposed solutions which can be now considered as classical in terms of their time of usage. But this problem continues to generate discussions among scholars because everybody wants to propose a new solution aiming at surpassing the performance of the previous solutions.

[6] points out that the increase of train speed further aggravates the problem of vehicle guiding force presence on curved surfaces. These authors propose the implementation of asymmetric profiles in order to solve this problem.

In his doctoral thesis, [7] has concluded that the majority of proposed solutions for train driving forces fall within the category of designing different patterns of rail curve lubricants. Indeed, research of several authors, such as [8,3], differs only in terms of lubrication type, application distance and other patterns.

\section{Methodology}

\subsection{Symbolism framework}

In this paper, several symbols will be used according to the meanings given below

$d_{\mathrm{v}}$ - Vertical wheel displacement in reference to profile; $\psi$ - The angle of displacement of wheels in reference to their radial position;

$\dot{\eta}_{\mathrm{w}}$ - Speed of lateral displacement of vehicle wheels;

$\dot{\eta}_{\mathrm{p}}$ - Speed of lateral displacement of profiles;

$d_{\mathrm{h}}-$ Horizontal (longitudinal) wheel displacement in reference to profile;

$\Delta \mathrm{r}$ - Marginal value of rolling circle radius that occurred during lateral displacement of wheels;

$\mathrm{r}_{\mathrm{a}}$ - Average value of rolling circle radius;

$\rho$ - Value of the bending level of profile under vehicle wheels couple;

$\psi$ - Velocity of wheel movement in the horizontal plane;

bs - Half of the distance between the circles of wheels rolling.

\subsection{Theoretical apparatus}

As previously explained in the problem identification section, the classical formulation of the guiding forces emerging during wheel-profile contact is characterized by both lateral (horizontal) guiding force and vertical force, as represented in Figure 1.

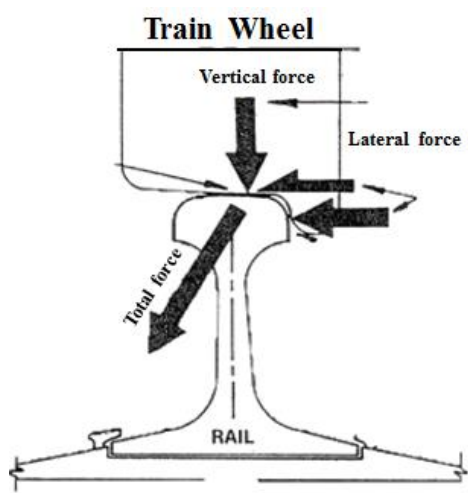

Figure 1. Graphical representation of guiding forces on wheel-profile contact

The indicator of wheel displacement will be used to describe the situation above in terms of the two components of the total driving force resulting in wheel-profile contact.

Analytically, Figure 1 is represented by equations (1) and (2) as explained in the paragraphs below.

On the one hand, vertical wheel displacement in reference to the profile is determined by the equation:

$$
d_{w}=\psi-\frac{1}{V}\left(\tilde{\eta}_{w}-\tilde{\eta}_{p}\right)
$$

On the other hand, horizontal (longitudinal) wheel displacement in reference to the profile is determined by the equation:

$$
d_{h}=\Delta r * r_{a}^{-1}+b_{a}\left(\rho-\tilde{\psi} * V^{-1}\right) .
$$

What can be concluded from the equations above is that vertical wheel displacement is mainly determined by $\psi-$ the angle of displacement of wheels in reference 
to their radial position ${ }^{2}$; while longitudinal wheel displacement in reference to the profile is mainly determined by the rotation angle of the pair of wheels.

Of course, all the abovementioned logic and analyses are applicable to tight curve surfaces.

In the case of a nonlinear trajectory, the angular and longitudinal relationship and the frictional force of all wheel couples cause the forming of additional moments, which increase the guiding forces. Therefore, this paper will describe vehicle movement in curves in terms of (1) displacement, and (2) moments and guiding forces.

\subsection{Data entry and analysis}

This paper will follow a simulation methodology for data entry and analysis.

For experimental purposes, a two-step approach is implemented. The two experimental stages were characterized by profiles designed following a curved path with a radius of $500 \mathrm{~m}$ (by definition classified as a tight curve).

The first experimental stage used standard profiles, while the second experimental stage exploited curvilinear profiles.

After the experiment was conducted, data concerning (1) displacement, (2) moments of force, and (3) guiding forces of wheel-rail contact were analyzed and compared for both stages both for standard and curvilinear profiles

\section{RESULTS AND DISCUSSIONS}

\subsection{Wheel displacement}

Figure 2 presents the results for both stages of the experiment (standard and curvilinear profiles) in terms of wheel displacement of the first and second pairs of wheels. As previously presented, both profiles are designed according to a curve with a radius of $500 \mathrm{~m}$.

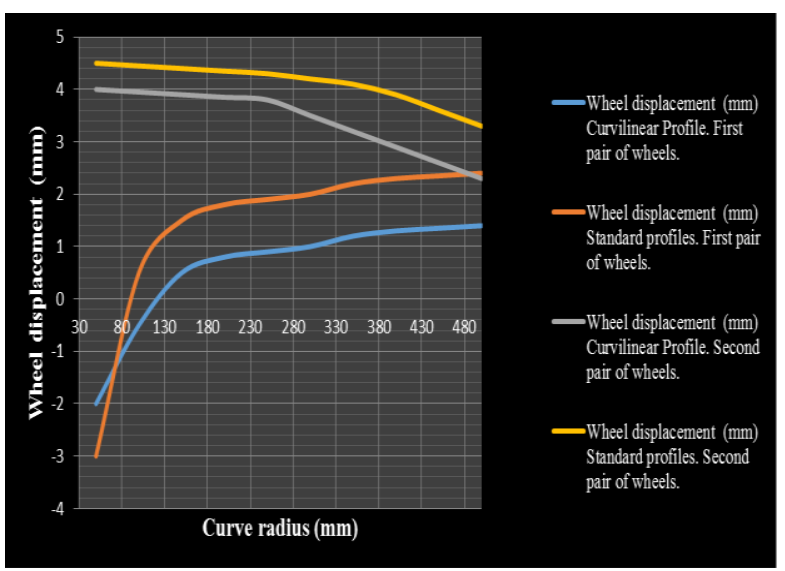

Figure 2. Wheel displacement of pairs of wheels - (a) first pair of wheels, (b) second pair of wheels
This graph confirms that using curvilinear profiles reduces wheel displacement. This lowers friction forces, thus influencing the reduction of wear.

By applying equation (1) and (2), the graph above suggests that in the case of curvilinear profiles, small movements of the vehicle lead to a change in the size of the wheel displacement smaller than the corresponding change in standard profiles.

\subsection{Moment of Forces}

Figure 3 presents results for both stages of the experiment (standard and curvilinear profiles) in terms of force moments of the first and second pairs of wheels.

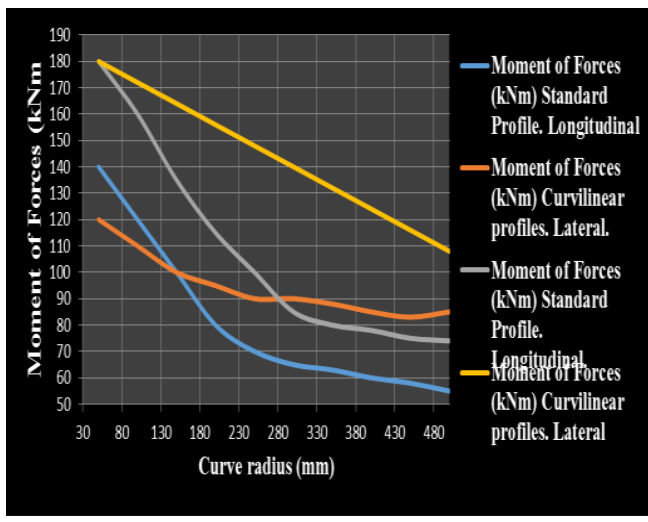

Figure 3. Moment of forces: (a) longitudinal forces, (b) lateral forces

As may be observed from the graph above, moments of force are greater in the case of standard profiles as compared to curvilinear ones. This is a consequence of the fact that the need for sufficient space for the movement of wheels on curvilinear profiles is greater than in standard ones.

This experiment confirmed that in curvilinear profiles with the increasing radius of the curve, moment of forces in the longitudinal direction in curved trajectories, significantly contributes to the reduction of the moment in comparison to standard profiles.

\subsection{Guiding forces}

Figure 4 shows results for both stages of the experiment (standard and curvilinear profiles) in terms of total guiding forces.

This is the most important indicator of the paper. Looking at the graph above, it can be stated that curvilinear profiles enable movement without many contact points with the wheel.

This is because in strong curves, as was previously tested, friction forces exert their action in a longitudinal direction by lowering the value of guiding forces.

In contrast, a standard profile is characterized by two contact points even in curved sections.

${ }^{2}$ Reduced by usually low value of $\frac{1}{V}\left(\dot{\eta}_{w}-\dot{\eta}_{p}\right)$ 


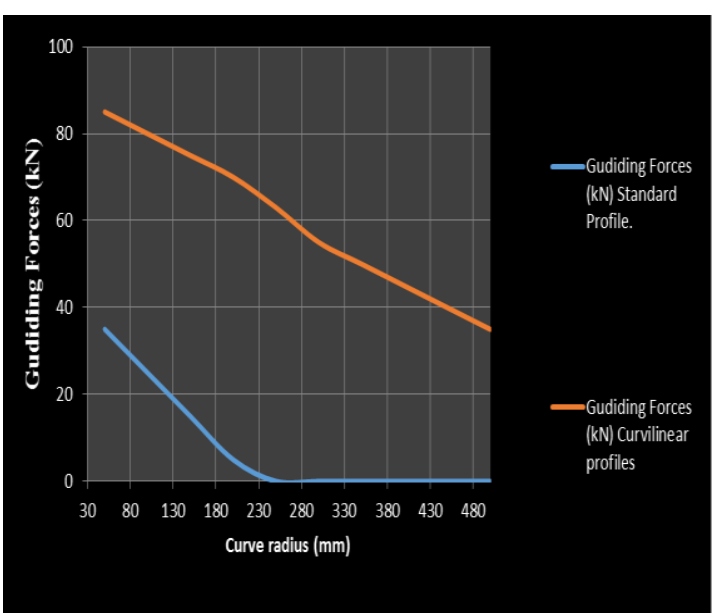

Figure 4. Guiding forces

\section{CONCLUSIONS}

This paper has tested the influence of the profile type on wheel-profile contact patterns. After experimental simulation, this research analyzed whether curvilinear profiles can impact: (1) displacement (2) moments of force and (3) guiding forces of the wheel-rail contact as compared to standard profiles.

The major conclusions drawn from the experimental results are: (1) in the case of curvilinear profiles, small movements of the vehicle lead to a change in the size of the wheel displacement smaller than the corresponding change in standard profiles; (2) moments of force are greater in the case of standard profiles compared to curvilinear ones; and (3) curvilinear profiles enable movement without many contact points with the wheel, while friction forces exert their action in a longitudinal direction, thus causing a smaller value of guiding forces.

The usage of curvilinear profiles causes a redistribution of forces and friction vectors, thus allowing a change (1) in friction and (2) lateral forces.

Given that these changes derived from using curvilinear profiles impact wear level, this paper's results impact the optimization of wheel-profile contact in curved segments.

\section{REFERENCES}

1. Railway applications - Testing and Simulation for the acceptance of running characteristics of railway vehicles
- Running Behaviour and stationary tests, EN 14363, Oct. 15, 2015.

Retrieved from:

https://shop.austrian-

standards.at/Preview.action;jsessionid $=85$ F8BFFD9F794 97A3EC8CC846B181177?preview $=\&$ dokkey $=583227 \&$ sele ctedLocale $=$ en

Retrieved on: Dec. 15, 2016

2. Testing and approval of railway vehicles from the point of view of their dynamic behavior - Safety - Track fatigue - Ride quality, 3rd ed., International Union of Railways, Paris, France, 2005.

Retrieved from:

http://xa.yimg.com/kq/groups/22520649/1117459065/n ame/uic

Retrieved on: Dec. 15, 2016

3. S. Kalay, R. P. Reiff, R. Smith, M. Scholl, "Control of Wheel/Rail friction," National Research Council, Washington (DC), USA, Rep, 71b, 2002.

Retrieved from:

http://onlinepubs.trb.org/onlinepubs/tcrp/tcrp rpt $71 \mathrm{~b}$ pdf

Retrieved on: Dec. 15, 2016

4. P. Dupont, "Testing the dynamic behavior of vehicles: Normalisation of test conditions by use of multi linear regressions," in Proc. WCRR 2011, Lille, France, 2011. Retrieved from:

http://www.railway-

research.org/IMG/pdf/g8 dupont patrick.pdf Retrieved on: Dec. 15, 2016

5. J. Wang, S. Chen, X. Li, Y. Wu, "Optimal rail profile design for a curved segment of a heavy haul railway using a response surface approach," Proc. Inst. Mech. Eng. Part F: J. Rail and Rapid Transit, vol. 230, no. 6, pp. 1496 1508, Aug. 2016.

DOI: 10.1177/0954409715602513

6. W. Zhai, J. Gao, P. Liu, K. Wang, "Reducing rail side wear on heavy-haul railway curves based on wheel-rail dynamic interaction," Int. J. Vehicle Mech. Mobility, vol. 52, no. suppl. 1, pp. 440 - 454, May 2014.

DOI: $10.1080 / 00423114.2014 .906633$

7. L. J. Wilson, "Performance measurements of rail curve lubricants," Ph.D. dissertation, School of Engineering Systems Queensland University of Technology, QUT, Brisbane, Australia, 2006.

Retrieved from:

https://eprints.qut.edu.au/16344/1/Lance Wilson Thesi s.pdf

Retrieved on: Dec. 15, 2016

8. S. Fukagai, T. Ban et al., "Development of Friction Moderating Systems to Improve Wheel/Rail Interface in Sharp Curves," in Proc. WCRR 2008, Seoul, Korea, 2008. Retrieved from:

http://uic.org/cdrom/2008/11 wcrr2008/pdf/S.2.2.4.2. pdf

Retrieved on: Dec. 15, 2016 